\title{
Effects of delay of prospective memory cues in an ongoing task on prospective memory task performance
}

\author{
Dawn M. McBride • Jaclyn K. Beckner • Drew H. Abney
}

Published online: 11 May 2011

(C) Psychonomic Society, Inc. 2011

\begin{abstract}
To address the mixed results reported in previous studies, the present experiments examined forgetting in prospective memory (PM) by manipulating the delay between the PM instructions and cue presentation in event-based PM tasks. PM performance was measured for delays of 2-20 min in Experiment 1 and for delays of approximately 1-10 $\mathrm{min}$ in Experiment 2. Experiment 2 included both focal and nonfocal PM tasks, and speed on the ongoing task was measured to examine evidence for monitoring processes across the delays tested. The results suggest that nonfocal PM performance follows a nonlinear forgetting function (i.e., rapid decline for shorter delays and slower decline for longer delays) when tested over delays from 1 to $20 \mathrm{~min}$. No effect of delay was seen for the focal task tested in Experiment 2 from 1 to $10 \mathrm{~min}$. In Experiment 2, ongoing-task costs were also found for the first delay but not for longer delays, suggesting that monitoring was significantly reduced between 1 and $2.5 \mathrm{~min}$ of the ongoing-task trials.
\end{abstract}

\section{Keyword Memory}

Forgetting in retrospective, explicit memory tasks has been well studied, with over 100 years of research indicating a characteristic function that shows rapid decline of performance for the shortest delays and slower declines in performance for longer delays (Rubin \& Wenzel, 1996). Forgetting characteristics of other forms of memory, however, are not as well understood. For example, in

D. M. McBride $(\bowtie) \cdot J$. K. Beckner • D. H. Abney

Department of Psychology Campus Box 4620,

Illinois State University,

Normal, IL 61790-4620, USA

e-mail:dmcbride@ilstu.edu prospective memory (PM), the effect of delay on task performance has been less clear.

PM has been defined as remembering to perform a task in the future (Einstein \& McDaniel, 1990). In a typical PM study, subjects are asked to remember to complete a future task (e.g., respond to a particular target word) while also completing an ongoing task (e.g., category judgments). Studies that have examined the effect of delay on PM-task performance have produced inconsistent results, with some studies showing a decline in performance for longer delays (e.g., Meier, Zimmermann, \& Perrig, 2006), and other studies showing no effect of delay on performance (e.g., Einstein, Holland, McDaniel, \& Guynn, 1992; Nigro \& Cicogna, 2000).

\section{Delay effects in PM tasks}

The majority of studies examining delay in PM tasks have manipulated delay by changing the length of an intervening task between the PM task instructions and the trials of the ongoing task in which PM cues are embedded. For example, Meier et al. (2006) asked subjects to complete an unrelated questionnaire for different lengths of time after being instructed to complete ongoing short-term memory trials, with a PM task to respond to certain types of items in the trials. Subjects began the short-term memory trials after the questionnaire task delay without mention of the PM task. Meier et al.'s results indicated that PM performance declined with longer delays.

An alternative procedure for examining delay in PM tasks is to manipulate the placement of the PM cues in the ongoing task. In Experiment 1 of their study, Brandimonte and Passolunghi (1994) examined delay in PM tasks in this manner and found that the first few minutes may be vital to 
remembering to perform the PM task. In their study, subjects were presented with several blocks of a short-term memory task that contained PM cues either immediately after the instructions were given or after a 3-min delay, during which subjects completed a block of short-term memory trials without presentation of the PM cues. Results indicated that PM-task performance declined from the immediate condition to the 3-min delay condition, suggesting that PM forgetting does take place in this time frame.

One interpretation of Brandimonte and Passolunghi's (1994) results is that subjects cannot maintain active monitoring for PM cues beyond a few minutes' time. Monitoring is one of the processes purported to aid in PMtask performance (Einstein \& McDaniel, 2010; Smith, 2010). If monitoring is needed for completion of PM tasks yet is difficult to maintain for long periods of time, then PM performance should decline with longer delays. Thus, knowledge regarding the maintenance of monitoring across an ongoing task may be informative in understanding PM forgetting across the length of an ongoing task.

Einstein et al. (2005, Exp. 2) attempted to address the issue of monitoring across the ongoing task by manipulating the type of PM task that subjects completed for PM cues placed in specific trials of the ongoing task. They argued (see also Einstein \& McDaniel, 2005) that less monitoring is needed when the PM task is focal to the ongoing task. This occurs when the ongoing task involves processing that allows the subject to focus on the relevant aspects of the PM cues. For example, if the PM cue is a specific word, the use of lexical decision or category identification ongoing tasks would create focal PM tasks, because these ongoing tasks allow the subject to process the target word as part of the task. Monitoring is more likely when the PM task is nonfocal, such that the relevant aspects of the PM cues are not processed as part of the task. For example, if the subject is asked to respond to words with a specific feature (e.g., a specific syllable, letter combination, or starting letter), lexical decision and category judgment ongoing tasks would not encourage processing of these features of the PM cues during the task. In the experiment conducted by Einstein et al. (2005), PM cues were included on the 40th, 80th, 120th, and 160th trials of a word categorization task. The PM task was to respond to two target words in the ongoing task (focal task) or to respond to words that contained the syllable "tor" (nonfocal task). Their results indicated that PM performance declined across trials (40th to 160th) for the nonfocal task, in which monitoring may have been difficult to maintain across the length of the task, but not for the focal task, in which monitoring may not have been needed to complete the PM task. It should also be noted that the length of the ongoing trials in the Einstein et al. (2005) study (based on the mean reaction times [RTs] reported for the ongoing task) was comparable to the time delay ( $3 \mathrm{~min}$ ) examined in the
Brandimonte and Passolunghi (1994) study. Taken together, the results of these studies indicate that monitoring for PM cues may decline within the first few minutes after PM instructions are given.

A set of studies conducted by Loft and colleagues (Loft, Kearney, \& Remington, 2008; Loft \& Yeo, 2007) also addressed the issue of monitoring for PM cues across the ongoing task. Loft et al. (2008) examined ongoing-task interference that occurred due to the PM task. RTs for ongoing-task trials were compared across a baseline block of ongoing-task trials without the PM task and a block of ongoing trials with the PM task included (non-PM cue trials only). Slower RTs for the PM-task block than the baseline block indicate interference due to use of attentional resources for the PM task (see Smith, 2003) and may have indicate that subjects were monitoring for PM cues during the task. Interference in the PM-task block was compared across different conditions in the Loft et al. experiments. The primary comparison was for subjects who were presented with PM cues and those who were not presented with PM cues in the ongoing task. Both sets of subjects were instructed to perform the PM task, so a difference in interference across conditions would indicate a difference in the level of monitoring for cues across the ongoing task. Subjects responded to specific target words as PM cues in the lexical decision ongoing task. Thus, the PM task would be classified as focal according to Einstein and McDaniel's (2005) definition. The results of the Loft et al. experiments indicated less interference when the PM cues were not presented than when they were. Thus, less monitoring occurred for the subjects who did not receive PM cues. Interference was still present in the no-PM-cue conditions (compared with a control condition in which no PM instructions were given), however, indicating that monitoring still occurred in this condition, just at a lower level than when PM cues were presented. In Experiment 3 of their study, Loft et al. examined how monitoring changed over the course of the ongoing task by including a third block of trials without PM cues for all subjects. This experiment showed that monitoring decreased over the course of ongoing trials for subjects who did not receive PM cues in any block of the experiment and was lower than it was for subjects who received PM cues in the second-block trials. However, some monitoring still occurred for subjects who did not receive PM cues (as compared with the control condition, in which no PM task was given), even after 640 trials of the ongoing task, a delay that was approximately $20 \mathrm{~min}$ (based on average RTs for the task plus the 5-min filler task completed before the ongoing trials began). Loft and Yeo (2007) reported similar results in a study that manipulated the frequency of PM cue presentations. Less-frequent PM cue presentations resulted in less monitoring and lower PM performance. 
From the results of the past studies described above, it is not yet clear at what point in the course of the ongoing task monitoring for PM cues begins to decrease and what time course the decrease in monitoring might take. This question is relevant to understanding how forgetting occurs in PM tasks and how long monitoring for PM cues can realistically be maintained in everyday PM tasks, because monitoring likely contributes to PM performance in many tasks (Einstein \& McDaniel, 2005; Smith, 2003). Loft et al.'s (2008) results seem to indicate that monitoring will not completely cease, since they observed interference in the ongoing task after a 20-min delay for a focal PM task. Brandimonte and Passolunghi's (1994) and Einstein et al.'s (2005) studies suggested that a decline in monitoring occurs in the first few minutes after PM intentions are formed, resulting in lower PM performance for longer delays (especially for nonfocal tasks). Loft et al.'s results suggested that the decline in monitoring may occur across as much as $20 \mathrm{~min}$, but that monitoring will still be present after $20 \mathrm{~min}$, even without presentation of a PM cue.

Einstein and McDaniel (2010) recently argued that although monitoring may be present in a study (as evidenced by ongoing-task costs), it may not have been required to complete the PM task. They argued that subjects may have engaged in monitoring on some trials in such studies, resulting in slower performance as compared with baseline conditions. They noted that slower performance in PM-task blocks does not rule out the use of spontaneous retrieval (another process that may be involved in PM performance under certain conditions; see Einstein \& McDaniel, 2005) to complete the PM task on all PM trials. Comparisons of PMtask performance across conditions that show different levels of interference can indicate whether spontaneous retrieval or monitoring is primarily responsible for retrieval on some PMtask trials, because high performance in the absence of ongoing-task costs shows that monitoring is not the primary means by which subjects are completing the PM task and that another process is likely contributing to PM performance. Loft et al. (2008) were not able to compare PM performance across their conditions, because their no-PM-cue condition did not involve presentation of the PM cues. Further, Einstein and McDaniel (2010) pointed out that monitoring processes can be difficult to sustain over trials in the ongoing task (see also West \& Craik, 1999). Thus, the extent to which monitoring occurs across the duration of ongoing-task trials is unclear.

Further investigations of forgetting in PM tasks across the length of an ongoing task are needed to clarify the time course of forgetting in PM and to address the question of how long monitoring is maintained across an ongoing task. In one such study, (Scullin, McDaniel, Shelton, \& Lee, 2010) measured monitoring (as task interference) for focal and nonfocal PM tasks across sets of 100 lexical decision trials in a 511-trial ongoing task. The PM cue appeared on Trial 501 in the ongoing task (a delay of approximately 10 min, based on mean RTs reported for the ongoing task plus a 5-min filler task), and Scullin et al. were interested in the level of monitoring that occurred across the ongoing task without presentation of a PM cue until the end of the task. Their results indicated that interference occurred for the nonfocal task in both the first and last sets of 100 ongoing-task trials. However, no interference was found for the focal task for the first or last sets of trials, despite high PM-task performance in this condition. Thus, Scullin et al. concluded that monitoring occurs for the nonfocal task, but not for the focal task across ongoing-task trials. Their study did not specifically address the question of how long monitoring would be maintained beyond $5 \mathrm{~min}$ of the ongoing task. Subjects also received a 5-min filler task in their study between the PM instructions and the start of the ongoing task, making it less likely that they would monitor for PM cues from the start of the ongoing task. Further, interference was still found in the Scullin et al. study in the last set of trials for the nonfocal task (similar to Loft et al.'s, 2008 , results), but it is unclear whether the levels of monitoring were similar in the first and last sets of trials. In another study that examined monitoring for a focal task, Harrison and Einstein (2010) found no evidence of monitoring for the trials immediately preceding the PM cue (despite high PM performance) and a decline in performance across blocks of the ongoing task. Thus, the present study was designed, specifically, to further address the question of monitoring across the ongoing-task trials, as well as to address the more general question of what functional form PM forgetting might take, by comparing PM performance across delays in all experiments, as well as by examining interference across delays in Experiment 2, to determine the extent of monitoring that occurs across a 10-min ongoing task without an interfering filler task.

\section{The present study}

To further study the effect of delay on PM, in the present study we manipulated delay for laboratory, event-based PM tasks. To address the question of how long subjects will monitor for PM cues across an ongoing task, delay was manipulated by placement of the PM cues within the ongoing task. However, in the present study, delay was manipulated between subjects to avoid effects of previous PM-task retrievals that might have been present in Einstein et al.'s (2005) study. In addition, the second experiment in the present study compared focal and nonfocal PM tasks, because (as described above) Einstein and McDaniel (2005) argued that the focal/nonfocal task contrast should affect the level of monitoring for PM cues that occurs within the ongoing task. Thus, the present study 
addressed four primary research questions regarding delay effects in PM: (1) Does PM-task performance decline over a range of delays of 1-20 min when the retention interval is manipulated by placement of PM cues in the ongoing task? (2) If PM forgetting does occur, what is its form? Experiment 2 also addressed the following questions: (3) Does monitoring decrease for longer delays of PM cue presentation? and (4) If monitoring does decrease with longer delays, is this decrease similar for focal and nonfocal tasks?

In Experiment 1, subjects engaged in a nonfocal PM task. According to Einstein et al. (2005), they should monitor for PM cues in this case, but monitoring might be difficult to maintain for long periods of time. Retention intervals of 2$20 \mathrm{~min}$ were tested to determine whether PM forgetting might occur within this time range after PM instructions are given. In Experiment 2, we attempted to generalize the results of Experiment 1 to a different set of ongoing and PM tasks and to a slightly shorter time course. PM performance was measured for delays in Experiment 2 that were based on the number of ongoing-task trials that preceded the PM cue $(40,100,200,300$, or 400 trials), which approximated delays of 1-10 min. Monitoring was measured by the interference in the ongoing task caused by the PM task. Interference was determined by comparing RTs for a baseline block (no PM task) and a PM-task block of the ongoing trials. A control group of subjects was also included in Experiment 2 in order to measure practice effects across blocks (Smith, Hunt, McVay, \& McConnell, 2007). In addition, the focality of the PM task was manipulated for different groups of subjects to allow for a comparison of forgetting based on a hypothesized difference in monitoring that should take place for these tasks, as described by Einstein and McDaniel (2005).

\section{Experiment 1}

\section{Method}

Subjects A total of 187 undergraduate students participated in Experiment 1 for course credit. The subjects were randomly assigned to delay conditions such that 36-38 subjects completed the experiment in each of five delay conditions: 2, 5, 10, 15, and $20 \mathrm{~min}$. Subjects who could not accurately recall the PM task at the end of the experiment were replaced.

Design and materials The subjects were presented with a practice block of trials (which contained one PM practice cue) and 19 blocks of experimental trials. One of these 19 blocks contained the three PM cues. Each block contained 5-15 trials. For each trial, a still photo from a movie was presented on the left side of the screen. Photos were obtained from various websites, and all photos were sized to fit into the same space on the screen. Four movie title options, lettered "a" through "d," were presented on the right side of the screen. The correct movie title was always presented as one of the options. At the end of each block, subjects saw a message asking them to recall the last three movies they chose on the preceding three trials. Block lengths varied so that subjects could not anticipate when the recall task would occur.

The PM cue block contained three movie slide trials that showed either a vehicle or glasses, along with seven other movie slide trials. No other movie slides used in the experiment contained a vehicle or glasses. The PM cue block was placed in the experiment at different places in the program in order to manipulate the delay for PM cues to be approximately $2,5,10,15$, or $20 \mathrm{~min}$ from the beginning of the experimental trials.

Procedure The subjects were instructed to complete a movie identification task. They were asked to view each movie photo that appeared on the screen and to choose the correct title from the choices on the right side of the screen. They were asked to press the correct letter $(a-d)$ on the keyboard that matched their response. Subjects were also told to keep track of the movies they chose, because they would be interrupted in the movie task periodically to recall out loud the last three movies they had chosen. Researchers manually recorded recall responses on a recall sheet. Subjects were asked to complete the PM task during the movie identification task. They were asked to press the space bar for any movie slide they saw that contained either a vehicle or glasses. The practice block was then completed, which included seven movie slides, one of which contained a vehicle. The subjects were asked to recall their last three movie choices at the end of the practice block. The researcher asked subjects to repeat the instructions for the tasks before they began the experimental trials, and if the subject did not correctly respond to the PM cue in the practice trials, this was pointed out to him or her. Subjects then completed 19 blocks of trials, with one of these blocks containing PM cues. The PM cue block was identical for all subjects; it just appeared in a different position in the ongoingtask trial blocks.

Trial slides were presented for $5 \mathrm{~s}$. Slides automatically advanced, even if the subject had not yet responded. Pilot testing showed that subjects could easily respond to most trials within this time period. For the recall task, a message appeared on the screen asking subjects to recall out loud the last three movies they had chosen and to press the spacebar to begin the next block of trials when they were finished. Recall was not timed, but subjects were encouraged to complete the recall task within $15 \mathrm{~s}$. Four short breaks were built into the program at the ends of the 4th, 8th, 12th, and 16th blocks, to allow 
subjects a break from the working memory recall task. Breaks occurred within one block of each PM block in the experimental session. A message appeared on the screen asking subjects to take a short rest before the trials continued. The breaks were timed for $60 \mathrm{~s}$. At the end of the experiment, subjects were again asked to describe the tasks they had completed in the experiment. If subjects did not recall the PM task at this time, they were replaced in the experiment, and their data were not analyzed.

Results and discussion

The proportions of correct responses to the PM targets on either the PM cue trials or the trials immediately following the PM cue were calculated for each subject. Data from 6 subjects were removed from the analysis because they did not recall any movies on the recall task for 3 or more blocks in the experiment. Analyses were conducted on data for the remaining 181 subjects. The mean proportions correct by delay condition are shown in Table 1. A one-way ANOVA on these data indicated an effect of delay, $F(4,176)=3.23$, $p=.014$. Post hoc tests with Bonferroni correction indicated that the largest decline in performance occurred between the 2- and 15-min delay conditions. Analyses were also conducted for PM-task accuracy on the first PM cue only. Overall, performance was lower for the first PM cue than for the average of all three PM cues, and although performance did decline across delays $(M=.39$ for the 2min delay, $M=.23$ for the 20-min delay), the delay effect did not achieve significance for the first-PM-cue data, $F(4$, $191)=1.56, p=.187$. The power to detect an effect in this analysis was .78 for a medium effect size (as estimated by G*Power; Erdfelder, Faul, \& Buchner, 1996). This result may indicate that, at least in some cases, subjects may have been able to retrieve the PM task for the 2nd or 3rd PM cue when they had missed the 1st PM cue presented.

Because a delay effect was found in Experiment 1 for all PM cue data, functions were also fit to these data to determine whether the functions that typically describe retrospective memory forgetting could also describe PM forgetting. Power, log, exponential, and linear functions were fit to the data in Table 1. These functions were

Table 1 Mean proportions of correct responses to PM cues in Experiment 1

\begin{tabular}{lll}
\hline Delay & $\mathrm{M}$ & $\mathrm{SE}$ \\
\hline $2 \mathrm{~min}$ & .55 & .07 \\
$5 \mathrm{~min}$ & .46 & .06 \\
$10 \mathrm{~min}$ & .33 & .06 \\
$15 \mathrm{~min}$ & .27 & .06 \\
$20 \mathrm{~min}$ & .31 & .07 \\
\hline
\end{tabular}

identified by Rubin and Wenzel (1996) as functions commonly fit to the forgetting data in retrospective memory studies. The power and log functions fit the data best, with $R^{2} \mathrm{~S}=.94$ for both. Figure 1 displays the best-fit power function, because this is the function that is more commonly descriptive of forgetting in retrospective memory studies (Rubin \& Wenzel, 1996; Wixted \& Ebbesen, 1991). Thus, the results of Experiment 1 indicate that for delays of 2-20 min from the beginning of the ongoing task to the presentation of PM cues, (a) PM performance does decline with longer delays and (b) PM forgetting follows a curvilinear function, with large declines in performance for the shortest delays and slower declines for longer delays.

\section{Experiment 2}

Experiment 2 was conducted in an attempt to generalize the results of Experiment 1 to the ongoing and PM tasks used in past PM studies (e.g., Einstein et al., 2005). In addition, the RT cost due to the PM task was measured to examine monitoring across the delays tested. Delays of approximately 1-10 min (estimated from mean RTs for 40, 100, 200, 300, and 400 trials that preceded the PM cue in different delay conditions) were tested for a category judgment ongoing task. For the PM tasks, subjects were asked to respond to the word "moose" (focal task) or to words with repeated o's (nonfocal task) during the category judgment task. A single PM cue ("moose") was positioned within the trials for both task conditions according to the delay subjects were assigned to. The manipulation of PM task type allowed for a test of Einstein et al.'s (2005) prediction that less forgetting should occur for focal than for nonfocal tasks, because subjects do not need to rely on monitoring (which cannot be easily maintained over long delays) for focal tasks. A finding of less forgetting for focal than for nonfocal tasks in the absence of monitoring would

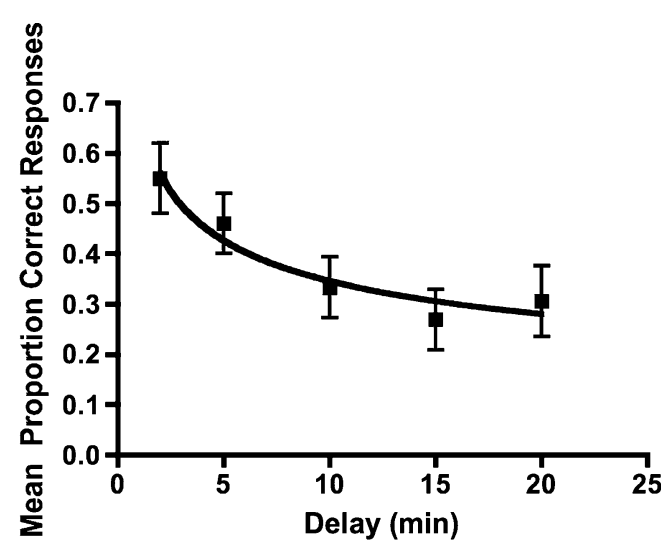

Fig. 1 Mean proportions of correct responses by delay for Experiment 1, with the best-fit power function. Error bars represent standard errors 
be consistent with Einstein et al.'s (2005) suggestion that spontaneous retrieval can account for PM performance in focal tasks.

\section{Method}

Subjects The subjects included 164 students at Illinois State University who volunteered to participate in the study. They were randomly assigned to 1 of the 10 delay/task conditions or to the control condition $(N=14$ per condition). Subjects who failed to follow instructions or otherwise indicated that they did not remember the PM task when questioned at the end of the experimental session were replaced in the experiment.

Design and materials The Delay (40, 100, 200, 300, and 400 trials before the PM cue presentation) and Task Type (focal and nonfocal) factors were manipulated between subjects. A control group was also included who completed two blocks of the ongoing task without the PM task in order to measure the practice effect that occurred across blocks of the experiment. All subjects completed 461 category judgment trials (60 trials in the baseline block and 401 trials in the PM block). Items were drawn from the Battig and Montague (1969) category norms. The same PM cue was used in all conditions. The word "moose" was chosen, because it served as a specific target word for the focal task condition and as a word with repeated o's for the nonfocal task condition. The word "moose" was only presented once in the experiment, and no other exemplars with repeated o's were presented. All PM-task subjects received the word "moose" with the category "Fruit." The category "Animal" was not used in the experiment. The PM cue appeared on the 41st, 101st, 201st, 301st, or 401st trial within the second block (PM block) of ongoing-task trials. The control subjects completed the second block of ongoing-task trials without the PM cue trial. Based on RT averages calculated for each delay/task condition, the total delays were found to be approximately $1,2.5,4.2,6.8$, and $10 \mathrm{~min}$.

Procedure The subjects were instructed that they would be completing a series of category judgment trials. Their task was to determine whether the exemplar on the right side of the screen belonged in the category given on the left side of the screen. Response keys on the keyboard were designated "yes" and "no." Although subjects were instructed to perform each category judgment quickly and accurately, trial slides were advanced only after the subjects responded. Subjects first performed a baseline block of 60 trials without the PM task. The first 20 trials within the baseline block were designated as practice trials and not included in the analyses. Subjects in the PM-task conditions were then instructed to complete another block of trials for the ongoing task along with the PM task. Subjects in the focal PM-task condition were instructed to press the space bar whenever they saw the word "moose" during the experiment. Those in the nonfocal PM-task condition were instructed to press the space bar whenever they saw a word with two consecutive o's. An example was given to clarify the nonfocal task. All subjects were asked to press the space bar as soon as they realized they had seen the instructed word or type of word and then to resume the category judgment task. Control subjects were simply asked to complete another block of trials for the ongoing task. Subjects who could not correctly recall the ongoing and PM tasks at the end of the experiment were replaced $(n=10)$.

Results and discussion

PM accuracy The proportion of subjects who correctly responded to the PM cue either during the PM cue trial or during the trial immediately following the PM cue was calculated for each Delay $\mathrm{x}$ Task Type condition. Responses that occurred outside of this time frame were not counted as correct (such responses occurred on $2.7 \%$ of the ongoing trials). Table 2 presents the mean accuracy performance on the PM task. An ANOVA with Delay and Task Type as between-subjects factors was conducted for these data. Both the main effect of delay, $F(4,130)=2.90$, $p=.025$, and the main effect of task type, $F(1,130)=$ $33.25, p<.001$, were significant in this analysis. The interaction between these factors was also significant, $F(4$, $130)=2.58, p=.040$. Simple effects analyses indicated that the effect of delay was significant for the nonfocal condition, $F(4,130)=4.88, p=.001$, but not for the focal condition, $F(4,130)=0.593, p=.668$. The power to detect a difference in performance across delays in the focal task was only .38 for a medium effect size (as estimated by G*Power; Erdfelder et al., 1996). Thus, it is possible that differences in performance across delays did exist in the focal task. However, an examination of the accuracy means in Table 2 shows that accuracy was highest (and at ceiling)

Table 2 Mean proportions of correct responses to PM cues by task type in Experiment 2 (standard errors are in parentheses)

\begin{tabular}{lll}
\hline Delay & Focal & Nonfocal \\
\hline $1 \mathrm{~min}$ & $.93(.11)$ & $.93(.11)$ \\
$2.5 \mathrm{~min}$ & $.93(.11)$ & $.43(.11)$ \\
$4.2 \mathrm{~min}$ & $.79(.11)$ & $.43(.11)$ \\
$6.8 \mathrm{~min}$ & $1.00(.11)$ & $.36(.11)$ \\
$10 \mathrm{~min}$ & $.86(.11)$ & $.43(.11)$ \\
\hline
\end{tabular}


for the 6.8-min delay condition and that accuracy did not decrease as delay increased. In contrast, accuracy means for the nonfocal task show a more consistent decline as delay increased. These means, coupled with the significant interaction, show a clear difference in performance across the focal and nonfocal tasks in Experiment 2.

As in Experiment 1, function fits were conducted for the mean data presented in Table 2 in order to describe the form of forgetting seen in the PM performance. Power, log, exponential, and linear functions were fit to the data for each task type separately. For the focal-task data, none of the functions fit the data very well; all provided $R^{2}$ values less than .10. These results further support the ANOVA results of little to no decline in performance across delay for the focal task. The exponential function provided the best fit to the nonfocal data, with $R^{2}=.98$. Figure 2 illustrates this best-fit function and the data points by delay for the nonfocal-task conditions.

Overall, the function fits are consistent with the results of Experiment 1: PM forgetting does occur with longer delays for nonfocal tasks, and this forgetting shows characteristic functions (power, exponential; see Rubin \& Wenzel, 1996) similar to those for forgetting seen in retrospective memory studies. These results are also consistent with Einstein et al.'s (2005) results showing forgetting only for the nonfocal task across shorter delays. The results for the focal task in Experiment 2 indicated little to no forgetting across delays of about $1-10 \mathrm{~min}$. This result is also predicted by Einstein et al.'s (2005) proposal that spontaneous retrieval of PM tasks can occur under focal-task conditions and can support high PM-task performance. Analysis of the RT difference scores will indicate whether the high performance for the focal conditions across delays was accompanied by a lack of monitoring over longer delays, as suggested by Einstein and McDaniel (2010).

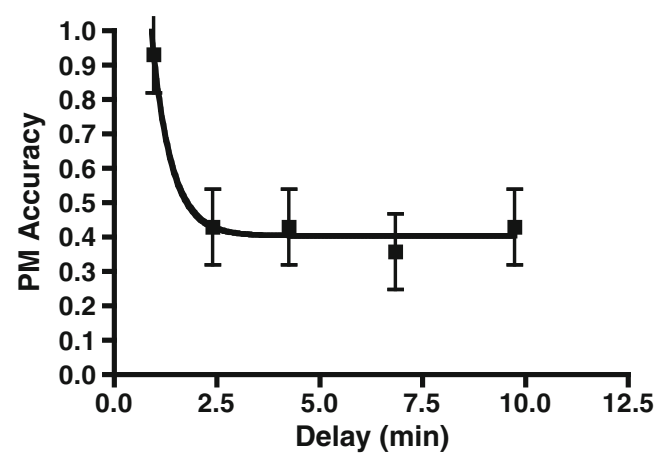

Fig. 2 Proportions of correct responses by delay for the nonfocal data of Experiment 2, with the best-fit exponential function. Error bars represent standard errors
$R T$ analyses The mean RT was calculated for each subject for the baseline block of ongoing trials without the PM task. The 20 practice trials that were presented at the beginning of this block were not included in this calculation. Thus, 40 baseline trials were included in the mean baseline RTs. The mean RT was also calculated for each subject for the 40 ongoing trials that preceded the PM cue, in order to determine the cost of including the PM task before presentation of the PM cue. This allowed us to determine whether monitoring occurred shortly before the PM cue was presented for each delay condition. In addition, this method of RT calculation allowed for comparable numbers of trials in the mean RTs for the baseline and PM blocks and across delay conditions. Trials that were three standard deviations from the subject's mean were excluded from the RT mean calculation ( $2.6 \%$ of all trials). To verify that baseline-block RTs for the PM subjects were consistent across conditions, a 2 (task type) x 5 (delay) ANOVA was conducted on the mean RTs for the baseline blocks alone; these RTs are shown in Table 3. Neither the main effects nor the interaction was significant for baseline-block RTs, all $p \mathrm{~s}>.05$. Thus, it was assumed that the baseline-block RTs were similar across conditions. Mean RT difference scores were then calculated for each subject by subtracting the baseline mean RT from the PM-block mean RT. Comparable blocks of trials were also examined for the control condition (i.e., the 40 trials that occurred in the same position in the 2nd block of ongoing trials for the control subjects), and difference scores comparable to each delay condition were also calculated for these subjects. All mean RT difference scores are presented in Table 4.

An ANOVA on mean RT difference scores was conducted with Delay and Task Type factors for the subjects who completed the PM task in Block 2 of the experiment. Both the main effect of delay, $F(4,130)=4.03$, $p=.004$, and the main effect of task type, $F(1,130)=5.28$, $p=.023$, were significant in this analysis. The interaction between these factors was not significant, $F(4,130)=1.24$, $p=.296$. The power for the interaction effect was estimated to be .965 , based on the effect size of $\eta_{\mathrm{p}}{ }^{2}=.13$ (a medium

Table 3 Mean RTs (in ms) for the baseline block in Experiment 2 by delay and PM-task conditions (standard errors are in parentheses)

\begin{tabular}{lll}
\hline Delay & Focal & Nonfocal \\
\hline $1 \mathrm{~min}$ & $1,335.36(67.14)$ & $1,233.39(32.27)$ \\
$2.5 \mathrm{~min}$ & $1,413.49(95.30)$ & $1,422.09(61.05)$ \\
$4.2 \mathrm{~min}$ & $1,337.30(57.22)$ & $1,337.18(75.99)$ \\
$6.8 \mathrm{~min}$ & $1,511.29(100.38)$ & $1,322.15(79.88)$ \\
$10 \mathrm{~min}$ & $1,549.00(63.01)$ & $1,394.38(95.38)$ \\
\hline
\end{tabular}


Table 4 Mean RT (in ms) differences (PM - baseline block) in Experiment 2 by delay and task conditions (standard errors are in parentheses)

\begin{tabular}{lrrr}
\hline Delay & \multicolumn{1}{l}{ Focal } & \multicolumn{1}{l}{ Nonfocal } & \multicolumn{1}{l}{ Control } \\
\hline $1 \mathrm{~min}$ & $68.92(35.92)$ & $277.01(72.82)$ & $-44.41(24.68)$ \\
$2.5 \mathrm{~min}$ & $-3.81(66.58)$ & $45.10(47.91)$ & $1.92(36.89)$ \\
$4.2 \mathrm{~min}$ & $-45.45(44.65)$ & $-79.01(103.37)$ & $-11.26(31.48)$ \\
$6.8 \mathrm{~min}$ & $-148.44(45.49)$ & $52.50(50.77)$ & $28.73(43.88)$ \\
$10 \mathrm{~min}$ & $-41.09(87.46)$ & $17.72(77.03)$ & $-31.55(32.26)$ \\
\hline
\end{tabular}

Negative values indicate faster performance in the second block (the PM block for PM-task conditions) of ongoing-task trials

to large effect size) for the comparable interaction effect (Task Type x Block) reported in Scullin et al. (2010, Exp. 3), using G*Power (Erdfelder et al., 1996).

Post hoc tests were conducted to compare delay conditions within the focal and nonfocal tasks. For the focal task, only one significant difference was found: The mean RT difference was higher for the 1-min delay condition than for the 6.8-min delay condition in the focal task, $p=.022$. No other significant differences were found for the focal task, all $p \mathrm{~s}>.127$. For the nonfocal task, the mean RT difference was higher for the 1-min delay condition than for all other delay conditions, all $p$ s $<.018$. All of the longer delays for the nonfocal task did not differ significantly, all $p \mathrm{~s}>.164$.

Mean RT differences were also compared for the PM-task conditions and the comparable control condition trials to determine whether the PM task caused significant slowing of the ongoing task for trials that immediately preceded the PM cues. For both the focal and nonfocal tasks, the mean RT difference was higher in the PM-task conditions than in the control condition for the 1-min delay, $p=.015$ and .001 , respectively, for the focal and nonfocal tasks. However, the PM-task condition mean RT differences were not significantly higher than the control mean RT differences for any other delay in either task, all $p \mathrm{~s}>.482$. In one condition (focal task, 6.8-min delay), the PM-task condition showed a significantly lower mean RT difference than the control condition, $p=.009$. These results indicate that monitoring occurred for both focal and nonfocal tasks before PM cue presentation at the earliest delay (about $1 \mathrm{~min}$ ), but decreased to nonsignificant levels for all longer delays (as compared with the control condition). If monitoring is the primary process by which PM tasks are accurately completed (see Smith, 2003), a reduction in PM-task accuracy would be expected to occur along with the decrease in monitoring after the earliest delay. This reduction in accuracy occurred for the nonfocal task, but not for the focal task. Thus, another process (e.g., spontaneous retrieval) must be the primary process supporting performance in the focal-task conditions for longer delays.

\section{General discussion}

The present study was designed to answer four questions regarding the effect of delay on PM performance in an event-based PM task: (1) Does PM-task performance decline over a range of delays from 1 to $20 \mathrm{~min}$ when retention intervals are manipulated without intervening tasks that might influence whether subjects engage in monitoring in the ongoing task? (2) If it occurs, what is the form of such forgetting? (3) Does monitoring decrease with longer delays of the PM cue in an ongoing task? and (4) If monitoring does decrease for longer delays, are the decreases similar for focal and nonfocal tasks? The results indicate that PM forgetting for nonfocal tasks does occur for delays in the range of 1-20 min, and that this forgetting follows a curvilinear function (power in Exp. 1, exponential in Exp. 2; see Rubin \& Wenzel, 1996). In other words, based on the results of the present study, PM performance for nonfocal tasks does decline with longer delays when the delays are filled with the ongoing task in which the PM task is embedded.

Function fits to the data indicated that curvilinear functions (power and exponential) fit the performance declines in the nonfocal data well, with functions accounting for $94 \%$ and $98 \%$ of the variance in the data in Experiments 1 and 2, respectively. Thus, for nonfocal PM tasks, the majority of forgetting occurs early in the ongoing task (in the first couple of minutes, in the present experiments), consistent with the time course proposed by Brandimonte and Passolunghi (1994). None of the functions fit the focal task data well in Experiment 2, because performance remained high across delays and showed little to no decline. In addition, forgetting results across the two experiments were fairly consistent, indicating similar declines in performance across delays for different sets of tasks.

Analyses of RT difference scores indicated that monitoring occurred only for the earliest delay in Experiment 2. For all longer delays, RT difference scores were similar across PMtask and control conditions (or were higher for the control condition, in the focal 6.8-min condition). These results occurred for both focal and nonfocal tasks; therefore, the type of PM task did not affect the time course of monitoring. Coupled with the accuracy results for focal and nonfocal tasks, the RT results indicate that monitoring declines across the ongoing task, decreasing accuracy in the nonfocal task, but not in the focal task. This pattern of results is consistent with suggestions made by Einstein and McDaniel (2010) that spontaneous retrieval can support performance in focal PM tasks. These results are also consistent with those reported by Scullin et al. (2010). In both studies, lower PM performance was found for nonfocal than for focal tasks at longer delays, with no evidence of monitoring in the focal task at the longer 
delays. Harrison and Einstein (2010) reported similar results for a focal task: high PM performance with no evidence of monitoring. Loft et al. (2008) also reported a decline in monitoring over the course of the ongoing task; however, they further reported that their subjects continued to monitor for about $20 \mathrm{~min}$ without presentation of the PM cue (similar to the timing of the PM cue presentation for the longer delays in the present experiments). In contrast, Experiment 2 of the present study showed no evidence of monitoring for longer delays up to $10 \mathrm{~min}$. The discrepancy in these results may be due to methodological differences across the studies that could affect the likelihood of monitoring on the part of the subjects. For example, the perceived PM task importance, the number of PM cues, the focality of the PM task, and task and experimental condition differences could all affect monitoring in the ongoing task (Einstein et al., 2005). The present study employed a different ongoing task, fewer PM cues, and different PM task instructions than the Loft et al. study, all of which might have affected the likelihood of monitoring across the two studies.

As described earlier, the majority of studies that have tested the effect of delays on PM-task performance have manipulated delay with an intervening task between PM instructions and the start of the ongoing task. In fact, many PM studies have included an intervening task as a means of reducing the likelihood of monitoring in the ongoing task for the PM cues. In the present study, no intervening task was included between the PM task instructions and the start of the ongoing task. This method was used to allow measurement of PM-task performance for very short delays (1-2 min) between the PM task instructions and the onset of PM cues. However, despite the lack of an intervening task, evidence for monitoring was only found for the shortest delay (1 $\mathrm{min})$ in Experiment 2.

In summary, the present study provides additional evidence that PM performance for nonfocal tasks declines with the delay between PM instructions and PM cue presentation in the ongoing task. Furthermore, these results indicated that nonfocal PM performance declines can be described with curvilinear functions of the sort typically fit to forgetting data in retrospective memory studies (power or exponential), illustrating a rapid decline in PM performance for short delays (1$5 \mathrm{~min}$ ) and a slower decline for longer delays (up to $20 \mathrm{~min}$ ). This result is inconsistent with results reported in past studies that PM performance does not decline across delays. It should be noted that the delays tested in the present study were relatively short compared to those that may exist for everyday PM tasks (e.g., hours or days). Thus, different forgetting characteristics may be found when longer delays are tested in future studies.
The results of the present study do suggest that monitoring for PM tasks (focal and nonfocal) declines in the first few minutes of the ongoing task. Coupled with the consistently high performance across delays for the focal task, these results support Einstein and McDaniel's (2005) suggestion that spontaneous retrieval can contribute to performance in focal PM tasks.

Author Note This research was supported by an Illinois State University Research Grant awarded to the first author. Members of the Human Memory Lab at Illinois State University (especially Kim French, Hannah Kibler, Stephanie Zegadlo, and Brandy Hucke) aided in data collection and discussion of the study results.

\section{References}

Battig, W. F., \& Montague, W. E. (1969). Category norms for verbal items in 56 categories: A replication and extension of the Connecticut category norms. Journal of Experimental Psychology Monographs, 80(3, Pt. 2), 1-46. doi:10.1037/h0027577.

Brandimonte, M. A., \& Passolunghi, M. C. (1994). The effect of cuefamiliarity, cue-distinctiveness, and retention interval on prospective remembering. The Quarterly Journal of Experimental Psychology, $47 A, 565-587$.

Einstein, G. O., Holland, L. J., McDaniel, M. A., \& Guynn, M. J. (1992). Age-related deficits in prospective memory: The influence of task complexity. Psychology and Aging, 7, 471-478.

Einstein, G. O., \& McDaniel, M. A. (1990). Normal aging and prospective memory. Journal of Experimental Psychology. Learning, Memory, and Cognition, 16, 717-726.

Einstein, G. O., \& McDaniel, M. A. (2005). Prospective memory: Multiple retrieval processes. Current Directions in Psychological Science, 14, 286-290.

Einstein, G. O., \& McDaniel, M. A. (2010). Prospective memory and what costs do not reveal about retrieval processes: A commentary on Smith, Hunt, McVay, and McConnell (2007). Journal of Experimental Psychology. Learning, Memory, and Cognition, 36, $1082-1088$.

Einstein, G. O., McDaniel, M. A., Thomas, R., Mayfield, S., Shank, H., Morisette, N., et al. (2005). Multiple processes in prospective memory retrieval: factors determining monitoring versus spontaneous retrieval. Journal of Experimental Psychology. General, 134, 327-342.

Erdfelder, E., Faul, F., \& Buchner, A. (1996). GPOWER: A general power analysis program. Behavior Research Methods, Instruments, \& Computers, 28, 1-11. doi:10.3758/BF03203630.

Harrison, T. L., \& Einstein, G. O. (2010). Prospective memory: Are preparatory attentional processes necessary for a single focal cue? Memory \& Cognition, 38, 860-867.

Loft, S., Kearney, R., \& Remington, R. (2008). Is task interference in event-based prospective memory dependent on cue presentation? Memory \& Cognition, 36, 139-148.

Loft, S., \& Yeo, G. (2007). An investigation into the resource requirements of event- based prospective memory. Memory \& Cognition, 35, 263-274.

Meier, B., Zimmermann, T. D., \& Perrig, W. J. (2006). Retrieval experience in prospective memory: Strategic monitoring and spontaneous retrieval. Memory, 14, 872-889. doi:10.1080/ 09658210600783774.

Nigro, G., \& Cicogna, P. C. (2000). Does delay affect prospective memory performance? European Psychologist, 5, 228-233. doi:10.1027/1016-9040.5.3.228. 
Rubin, D. C., \& Wenzel, A. E. (1996). One hundred years of forgetting: A quantitative description of retention. Psychological Review, 103, 734-760. doi:10.1037/0033-295X.103.4.734.

Scullin, M. K., McDaniel, M. A., Shelton, J. T., \& Lee, J. H. (2010). Focal/nonfocal cue effects in prospective memory: Monitoring difficulty or different retrieval processes? Journal of Experimental Psychology. Learning, Memory, and Cognition, 36, 736-749. doi:10.1037/a0018971.

Smith, R. E. (2003). The cost of remembering to remember in event-based prospective memory: Investigating the capacity demands of delayed intention performance. Journal of Experimental Psychology. Learning, Memory, and Cognition, 29, 347-361.
Smith, R. E. (2010). What costs do reveal and moving beyond the cost debate: Reply to Einstein and McDaniel. Journal of Experimental Psychology. Learning, Memory, and Cognition, 36, 1089-1095.

Smith, R. E., Hunt, R. R., McVay, J. C., \& McConnell, M. D. (2007). The cost of event-based prospective memory: Salient target events. Journal of Experimental Psychology. Learning, Memory, and Cognition, 33, 734-746.

West, R., \& Craik, F. I. M. (1999). Age-related decline in prospective memory: The roles of cue accessibility and cue sensitivity. Psychology and Aging, 14, 264-272.

Wixted, J. T., \& Ebbesen, E. B. (1991). On the form of forgetting. Psychological Science, 2, 409-415. 